\title{
GEOMETRICAL MODELING OF WOVEN FABRICS WEAVABILITY-LIMIT NEW RELATIONSHIPS
}

\author{
Mohamed Dalal', Jean-Yves Drean², Jean-François Osselin² \\ ${ }^{1}$ REMTEX research laboratory /Ecole Supérieure des Industries du Textile et de l'habillement - ESITH Route d'Eljadida, Km 8 - BP. 7731 Oulfa, Casablanca \\ Morocco; dalalmohamed@gmail.com - dalalmohamed@hotmail.com dalal@esith.ac.ma \\ ${ }^{2}$ LPMT research laboratory / Université de Haute Alsace (UHA), - Laboratoire de Physique et Mécanique Textiles (LPMT) EA 4365, 68093 Mulhouse, \\ France; jean-yves.drean@uha.fr - jean-francois.osselin@uha.fr
}

\begin{abstract}
:
The weavability limit and tightness for $2 D$ and $3 D$ woven fabrics is an important factor and depends on many geometric parameters. Based on a comprehensive review of the literature on textile fabric construction and property, and related research on fabric geometry, a study of the weavability limit and tightness relationships of $2 D$ and $3 D$ woven fabrics was undertaken. Experiments were conducted on a representative number of polyester and cotton woven fabrics which have been woven in our workshop, using three machines endowed with different insertion systems (rapier, projectiles and air jet). Afterwards, these woven fabrics have been analyzed in the laboratory to determine their physical and mechanical characteristics using air permeability-meter and KES-F KAWABATA Evaluation System for Fabrics. In this study, the current Booten's weavability limit and tightness relationships based on Ashenhurst's, Peirce's, Love's, Russell's, Galuszynskl's theory and maximum-weavability is reviewed and modified as new relationships to expand their use to general cases ( $2 D$ and $3 D$ woven fabrics, all fiber materiel, all yarns etc...). The theoretical relationships were examined and found to agree with experimental results. It was concluded that the weavability limit and tightness relationships are useful tools for weavers in predicting whether a proposed fabric construction was weavable and also in predicting and explaining their physical and mechanical properties.
\end{abstract}

\section{Keywords:}

$2 D$ and $3 D$ woven fabrics, weavability limit and tightness, physical and mechanical characteristic, modeling.

\section{Introduction}

During last many years, many works have conducted a considerable amount of research regarding the weavability limit, the maximum number of warps and picks per unit length that can be woven with a given yarn and weave. The driving force behind such research effort is: first, to avoid attempting to weave impossible constructions and thus eliminating unnecessary costly trials, and second, to reduce yarn breakage during weaving and wear of weaving-machine parts. For only simple woven fabrics, the early work focused on conducting experiments that led to empirical relationships [1-8] that correlated the maximum number of threads per unit length with yam size and weave. Also, more recent research has followed the same theoretical approaches [9-24] to broaden the applications of the weavability-limit relationships.

More recent and current research efforts have been directed toward establishing a standard or reference fabric against which fabrics can be compared. The reference fabric is normally that of maximum construction. The proposed comparison is usually expressed in terms of the ratio of a construction parameter of a given fabric to the corresponding value for the standard fabric. The ratio is termed firmness or tightness. The incentive behind such research is the thought that the physical, mechanical, and aesthetic properties of a fabric can be related to its degree of tightness. Designers would thus have scientific rules to construct fabrics with predetermined end-use performance. It was found that a wide variety of properties of woven fabrics depend on the degree of tightness [25-38].

Since most of the research on weavability limit and tightness has been published when natural fibers were still dominant, the derived and proposed relationships are limited to fabrics from wool or cotton fiber. This issue of Textile Progress reviews the soundest research work on the weavability limit, proposed tightness parameters, and the relationship between fabric properties and tightness. But all the relationship weavability limit and tightness published until nowadays are used only for simple woven fabrics and neither for complex woven fabrics nor for all material. And also does not give us true information about weavability limit and tightness.

Therefore, the objective of this new research is to establish a correct standard or reference fabric against which fabrics can be objectively 
compared. Furthermore, the weavability limit and tightness relationships reviewed are modified to expand their use to general cases (any weave simple or complex, fiber type, etc.). The theoretical relations were examined and found to agree with experimental results. It was concluded that the theoretical relations and the graphs derived from them were useful tools for weavers in predicting whether a proposed fabric construction was weavable.

\section{Definition and objective}

Following the same theoretical approaches, considering simple and complex woven fabrics and keeping in mind that we can never have straight warp and straight weft at the same time; we have established a correct standard or reference fabric against which fabrics can be objectively compared. Furthermore, the weavability limit and tightness relationships reviewed are modified to expand their use to general cases (any weave simple or complex, fiber type, etc.).

So in practice, we usually assimilate the limit of weavability to the maximum weft count, which can be reached in the optimum adjustment conditions of the machine, for a woven fabric, and using a predetermined weaving machine.

It is necessary to note that

- This limit can be determined only empirically and it requires a preliminary test for every woven fabric;

- The limit of weavability which corresponds to an acceptable rate of breakage and quality is lower to the technical limit.

For these reasons, it is interesting to design a mathematical model, based on the simplified structure of woven fabrics, which allows encoding the difficulty to weave a woven fabric with known pattern and thread count. The limit of the saturation index as defined, will constitute the limit of weavability whatever is the machine used.

The difficulty to weave a fabric can come from:

- The warp thread count

- The weft thread count

- Both thread counts

It is, therefore, necessary to consider each of these factors separately.

On the condition of defining a maximum warp count (respectively weft count) corresponding to a given linear density of the yarn, the indication of saturation can be expressed as follows:

- For the warp by the ratio of the warp account on the maximal warp account $=I s w p$

- For the weft, by the ratio of the weft count on the maximal weft count $=I s w f$

In order to facilitate its expressions, we provide the values in percent "\%" as follows:

$$
\begin{aligned}
& \text { Warp saturation index } \Rightarrow \begin{array}{l}
I s w p \%=100 \times \frac{\text { Cwarp }}{\text { Cwarp } \max } \\
\text { Weft saturation index }
\end{array} \Rightarrow I s w f \%=100 \times \frac{\text { Cweft }}{\text { Cweft } \max }
\end{aligned}
$$

And the difficulty of weavability is provided by a general indication (warp and weft):

$$
\text { Woven fabric saturation index } \Rightarrow I s w \%=\frac{I s w p \times I s w f}{100}
$$

\subsection{Determinations of Iswp, Iswf and Isw}

The warp count being known, it is enough to determine its maximal value. For that, it is necessary to make the 3 following assumptions:

Assumption 1: threads have a cylindrical cross-section [1-24]

Assumption 2: in the case of the most contracted thread count, threads are contiguous under floating and are separated only by a thread thickness (therefore of the value of a diameter, according to the "1st Assumption" at the time of face changes.

Assumption 3: the linear density and material density [g/cm3] are known and homogeneous. 


\section{Remarks:}

It is obvious that the structure of the face (Figure 1.a, b, c) can represent the warp thread and the weft thread at a given time because it is supposed that the crimp percentage and take-up percentage are simultaneously equal to zero, which is impossible.

In general the material areal density is known, but not the thread; therefore it is preferable to measure the diameter rather than to calculate it. And the best method to measuring the obvious diameter of the thread with sufficient statistical precision is by an optical method.

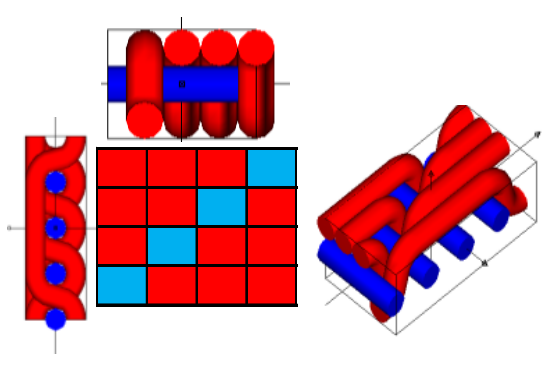

a : Simple Woven Fabric: Straight Weft Figure 1. Example of design

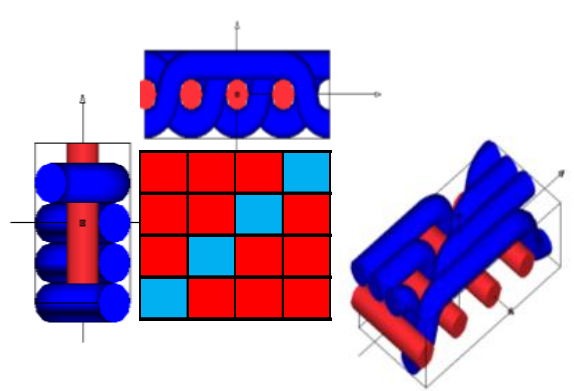

b : Simple Woven Fabric: Straight Warp

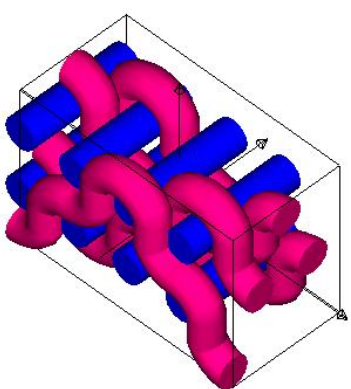

C : Warp Double-Faced Woven Fabric

Unfortunately, this method is time consuming and is more practical to calculate it from the linear density.

\subsection{Demonstration for simple woven fabrics " 1 Warp +1 Weft":}

From the geometry of the warp and weft cross-sections of a weave (Figure 2 and 3) and using the 3 assumptions made in developing the maximum set of woven fabrics, the general equations of the maximum set for warp "Cwp ${ }_{\max }$ " and weft "Cwf ${ }_{\max }$ " threads can be derived as:

First case: Straight Warp and Maximal Crimped Weft

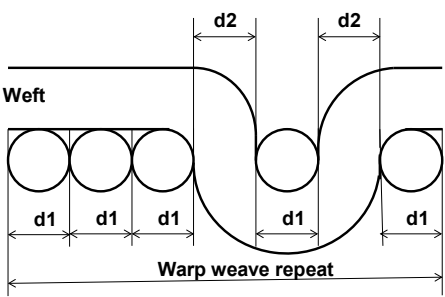

Warp cross section

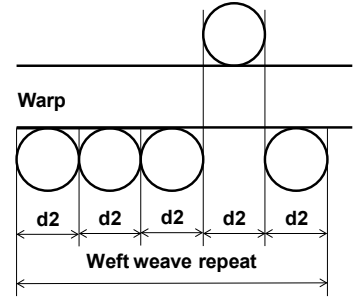

Weft cross section

$$
\mathrm{Cwp}_{\max }[\text { threads } / \mathrm{cm}]=\frac{\mathrm{Rwp}}{\mathrm{Rwp} \cdot d_{1}+\mathrm{Nwf} \cdot d_{2}}
$$

Figure 2. Maximum set of woven fabrics for straight warp

$$
\mathrm{Cwf}_{\max }[\text { threads } / \mathrm{cm}]=\frac{\mathrm{Rwf}}{\mathrm{Rwf} \times d_{2}}=\frac{1}{d_{2}}
$$

\section{Second case: Maximal Crimped Warp and Straight Weft}

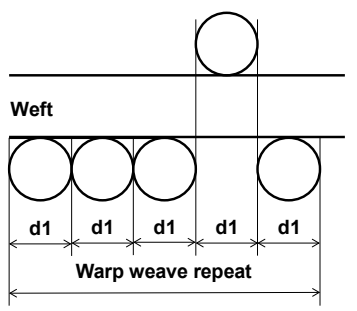

Warp cross section

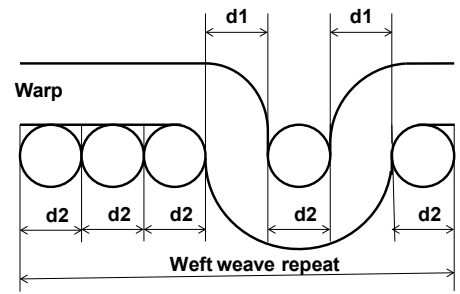

Weft cross section

$$
\mathrm{Cwp}_{\max }[\text { threads } / \mathrm{cm}]=\frac{\mathrm{Rwp}}{\mathrm{Rwp} \cdot d_{1}}=\frac{1}{d_{1}[\mathrm{~cm}]}
$$

$\mathrm{Cwf}_{\max }[$ threads $/ \mathrm{cm}]=\frac{\mathrm{Rwf}}{\mathrm{Rwf} \cdot d_{2}+\mathrm{Nwp} \cdot d_{1}}$

Figure 3. Maximum set of woven fabrics for straight Weft 
Using equations (1), (2), (3), (4), (5), (6), (7) and in order to generalize the saturation equations, we have introduced an additional parameter " $\alpha$ " as a coefficient which varies depending on the warp tension.

$$
\begin{array}{ccc}
\text { very high warp tension } & <\alpha< & \text { very low warp tension } \\
0 & <\alpha< & 1
\end{array}
$$

Because the saturation can be obtained either by weaving with a very high warp tension (Straight Warp and Maximal Crimped Weft) or with very a low warp tension (Maximal Crimped Warp and Straight Weft).

We can express the following relationship:

$$
\begin{gathered}
I s w p \%=K \times C w p \times\left(\sqrt{\frac{T w p}{\phi \rho_{f w p}}}+(1-\alpha) \frac{N w f}{R w p} \sqrt{\frac{T w f}{\phi \rho_{f w f}}}\right) \\
I s w f \%=K \times C w f \times\left(\sqrt{\frac{T w f}{\phi \rho_{f w f}}}+\alpha \frac{N w p}{R w f} \sqrt{\frac{T w p}{\phi \rho_{f w p}}}\right)
\end{gathered}
$$

With:

$$
\text { First case : } \alpha=0
$$

Straight Warp and Maximal Crimped Weft

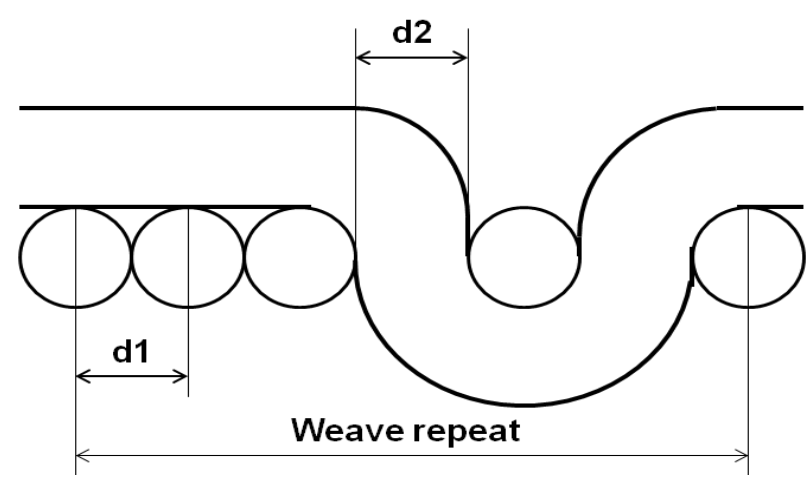

Warp cross section
Second case : $\alpha=1$

Maximal Crimped Warp and Straight Weft

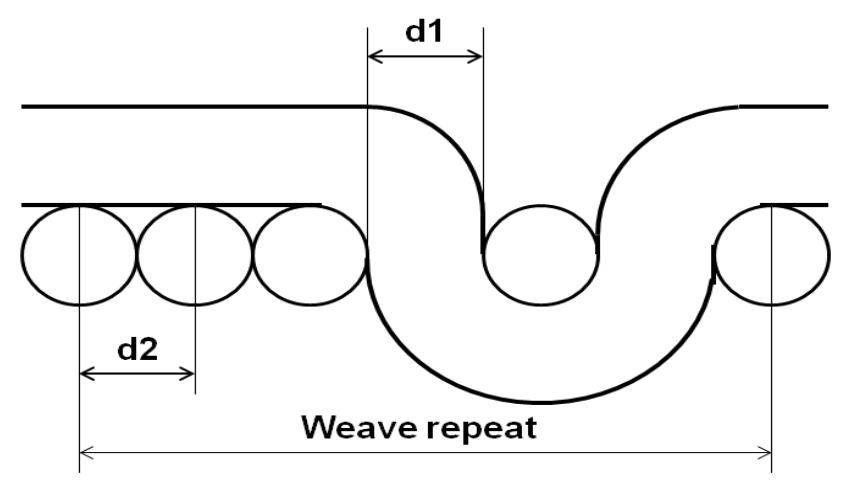

Weft cross section

we can never had straight warp and straight weft at the same time

Figure 4. Theory of geometry of the warp and weft cross-sections of a weave

Where:

a : Warp tension coefficient

d1 : warp-thread diameter [cm],

d2 : weft-thread diameter [cm],

Cwp : Warp count [threads/cm],

Cwf : Weft count [threads/cm],

Twp : Warp linear density [Tex],

Twf : Weft linear density [Tex],

Nwf : Weft number of face change by weave repeat,

Nwp : Warp number of face change by weave repeat,

Rwp : Warp repeat weave,

Rwf : Weft repeat weave,

if : fiber density [g/cm3],

$F \quad$ :yarn packing fraction,

Using same a way we can demonstrate that: 
For a 1.Warp + 1. Weft woven fabric, the warp and weft saturation index with $\alpha=0$ or $\alpha=1$ is as follows:

$$
\begin{aligned}
& I s w p \%=K \times C w p \times\left(\sqrt{\frac{T w p}{\phi \rho_{f w p}}}+(1-\alpha) \frac{N w f}{R w p} \sqrt{\frac{T w f}{\phi \rho_{f w f}}}\right) \\
& I s w f \%=K \times C w f \times\left(\sqrt{\frac{T w f}{\phi \rho_{f w f}}}+\alpha \frac{N w p}{R w f} \sqrt{\frac{T w p}{\phi \rho_{f w p}}}\right)
\end{aligned}
$$

For a 1 Warp $+n$ Wefts woven fabric, the warp and weft saturation index with $\alpha=1$ is as follows:

$$
\begin{aligned}
& I s w p \%=K \times C w p \times\left(\sqrt{\frac{T w p}{\phi \rho_{f w p}}}+\alpha \sum_{i=1}^{n} \frac{N w f_{n}}{R w p} \times \sqrt{\frac{T w f_{n}}{\phi \rho_{f w f_{n}}}}\right) \\
& I s w f \%=\operatorname{Sup}_{n}\left\{K \times P w f_{n} \times C w f \times\left(\sqrt{\frac{T w f_{n}}{\phi \rho_{f w f_{n}}}}+(1-\alpha) \frac{N w p}{R w f_{n}} \sqrt{\frac{T w p}{\phi \rho_{f w p}}}\right)\right\}
\end{aligned}
$$

For a $\mathrm{n}$ Warps +1 Wefts woven fabric, the warp and weft saturation index with $\alpha=0$ is as follows:

$$
\begin{aligned}
& I s w p \%=\operatorname{Sup}_{n}\left\{K \times P w p_{n} \times C w p \times\left(\sqrt{\frac{T w p_{n}}{\phi \rho_{f w p_{n}}}}+\alpha \frac{N w f}{R w p_{n}} \sqrt{\frac{T w f}{\phi \rho_{f w f}}}\right)\right\} \\
& I s w f \%=K \times C w f \times\left(\sqrt{\frac{T w f}{\phi \rho_{f w f}}}+(1-\alpha) \sum_{i=1}^{n} \frac{N w p_{n}}{R w f} \times \sqrt{\frac{T w p_{n}}{\phi \rho_{f w p_{n}}}}\right)
\end{aligned}
$$

Depending on the woven fabrics we use the combination of formulas obtained in cases of $n$.Warps $+n$.Wefts: The weave index remains the same as equation (01).

\begin{tabular}{|c|c|c|c|c|}
\hline Weaving machine & & Rapier & Air jet & Projectil \\
\hline Weaving speed & & 420 picks/min & 580 picks/min & 345 picks/min \\
\hline Warp & Yarn count & 26 yarns / cm & 24 yarns / cm & 24 yarns / cm \\
\hline \multicolumn{2}{|c|}{ material } & cotton & polyester & cotton \\
\hline & Linear density & 40 tex & 300 dtex & 50 tex \\
\hline \multirow{3}{*}{ Weft } & Yarn count & $\geq 10$ picks $/ \mathrm{cm}$ & $\geq 10$ picks $/ \mathrm{cm}$ & $\geq 10$ picks $/ \mathrm{cm}$ \\
\hline & material & Cotton and polyester & Cotton and polyester & Cotton and polyester \\
\hline & Linear density & variable & variable & variable \\
\hline Drawing-in width & & $175 \mathrm{~cm}$ & $180 \mathrm{~cm}$ & $184 \mathrm{~cm}$ \\
\hline
\end{tabular}

$$
I s w \%=\frac{I s w p \times I s w f}{100}
$$

\section{Experimental procedure}

\subsection{Materials}

Table 1 compiles the information related to the rapier, the air jet and the projectile weaving machines.

Table 1. Rapier weaving machine

The weft cotton count is $10,14,18,22,26,30,34,40,46$ and 50 tex and weft polyester count 150, 200, 250, 300, 350, 400, 450 and 600 dtex 
Parameters:

Stationary parameters

Variable parameters (weft)

Variable parameters (warp)

Variable parameters (pattern)
: warps and its characteristics

: weft count, titres of weft and material

: warp tension to insert picks / cm

$:(n$ Warps +1 Weft $)$ and (1 Warp $+n$ Wefts)

\subsection{Procedure}

We have produced a representative number of simple and complex patterns with different qualities of weft while increasing weft count and warp tension until saturation of woven fabrics.

\section{Results and discussion}

If the formulated hypotheses are respected, the warp saturation index or weft saturation index is reached when the warp count or weft count is respectively equal to the maximum warp count or maximum weft count.

$$
\begin{gathered}
\text { Warp saturation index } \Rightarrow \text { Iswp }=100 \% \\
\text { Or } \\
\text { Weft Saturation index } \Rightarrow \text { Iswf }=100 \%
\end{gathered}
$$

But the given hypotheses are not entirely true. Indeed:

- The diameter of thread is not cylindrical; in particular, threads partially flatten during weaving process(criss-crosses);

- In the most contracted thread count, we may have partial overlap of threads and distortion of these (modification of the obvious diameter),

- The crimp percentage and the take-up percentage are not practically equal to zero in woven fabrics;

- Material density depends on many factors as materiel, curliness, type of spinning, etc.

Therefore it is quite normal that the practical limit of weavability is different from the theoretical limits.

The abacus (Figure 5) can be used to choice warp and weft characteristic function of the degree of saturation index we want and also to avoid the unnecessary cost of production while trying to weave saturated contextures. For a given pattern and known warp characteristics, we can predict the weft characteristics and contexture according to the saturation index desired. We can also make the combinations between warp characteristics, weft characteristics and pattern to anticipate the difficulties of weaving using the value of saturation index.

\subsection{Validation of the Weavability-limit relationships for Simple weaves}

The experiments (Figure 6), that we conducted, showed that the practical limit of weavability for simple woven fabric is at $70 \%$ $( \pm 5 \%)$ of Woven fabric Saturation index

And Very good correlation (Figure 7) is observed between practical and theoretical maximal weft count for simple weaves. The practical weft count is obtained by experience at the weavability limit using different looms and the theoretical maximal weft count is obtained by using the availability-limit relationships.

\subsection{Validation of the Weavability-limit relationships for complex weaves}

The experiments (Figure 8), that we conducted, showed that the practical limit of weavability for complex woven fabric is at $70 \%$ $( \pm 5 \%)$ of Woven fabric Saturation index. 


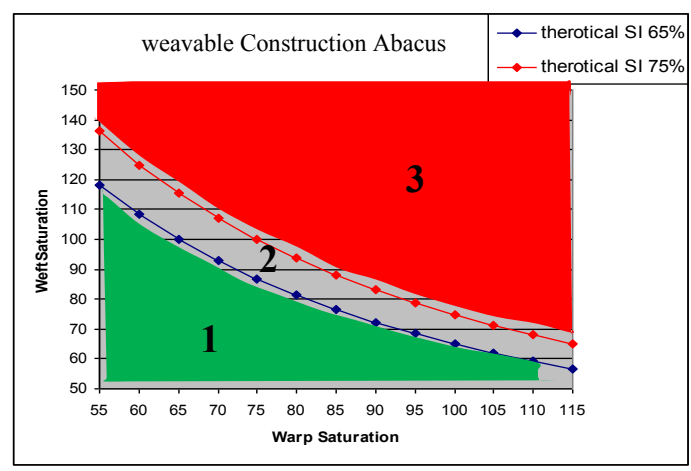

1: Classical and Technical construction

2: Technical woven fabrics construction

3: Impossible woven fabrics construction

Figure 5. weavable Construction Abacus

Rapier weaving machine-Polyester Weft

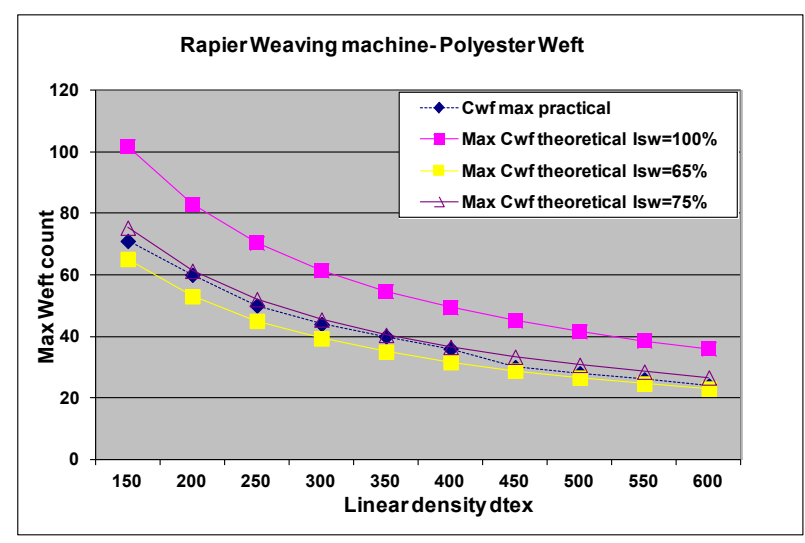

Projectile weaving machine-Polyester Weft

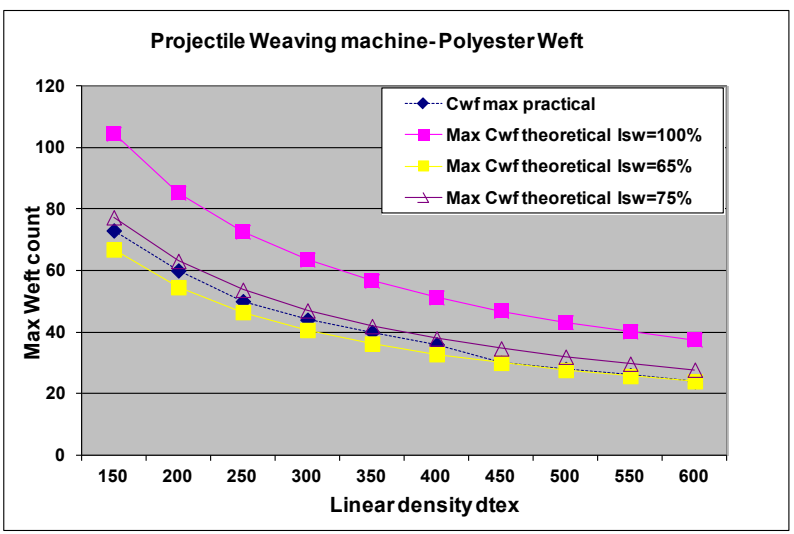

Air jet weaving machine-Polyester Weft

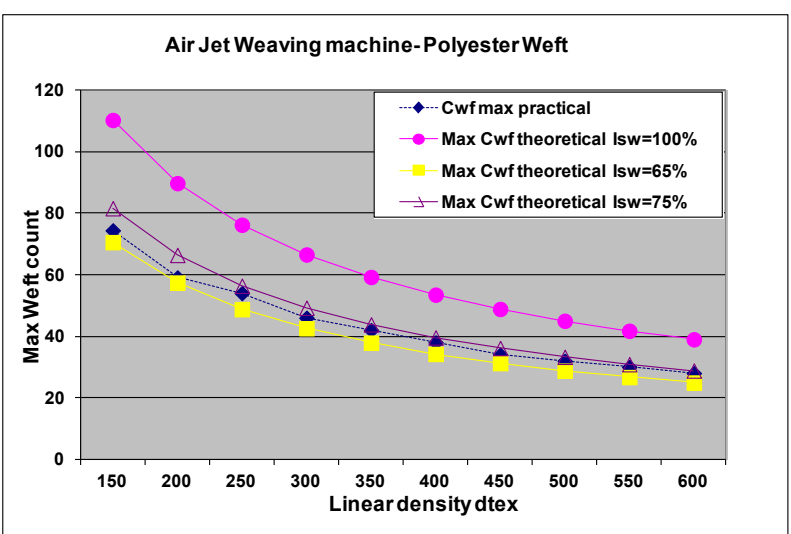

Rapier weaving machine-Cotton Weft

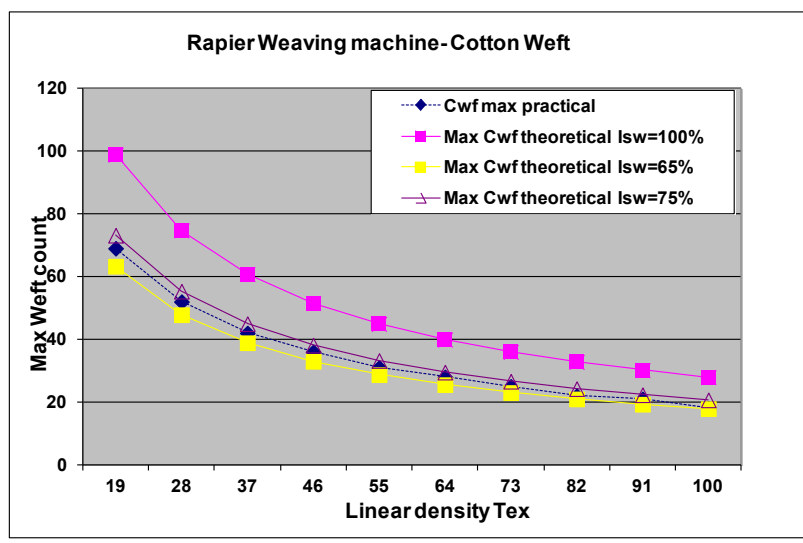

Projectile weaving machine-Cotton Weft

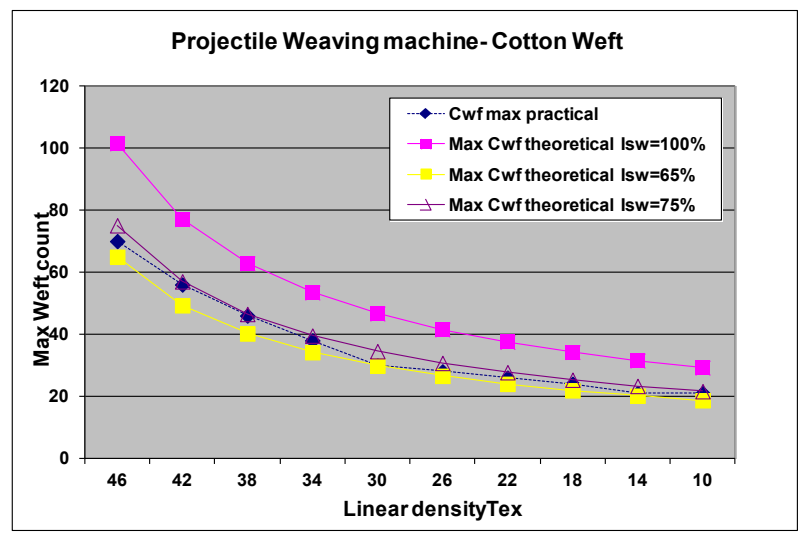

Air jet weaving machine-Cotton Weft

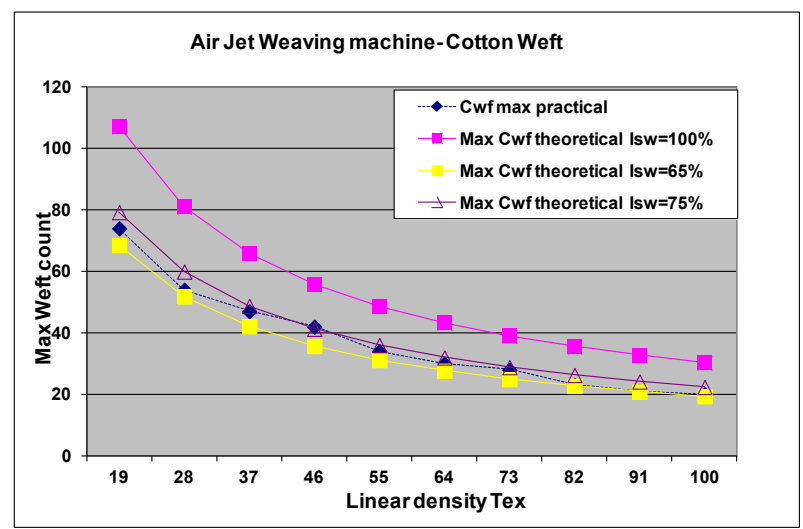

Figure 6. Validation of the Weavability-limit relationships for Simple weaves 
Rapier weaving machine-Polyester Weft

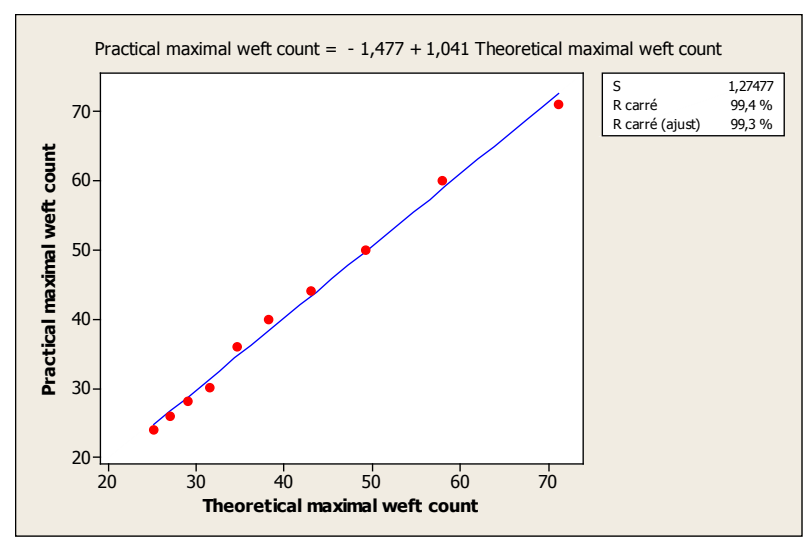

Projectile weaving machine-Polyester Weft

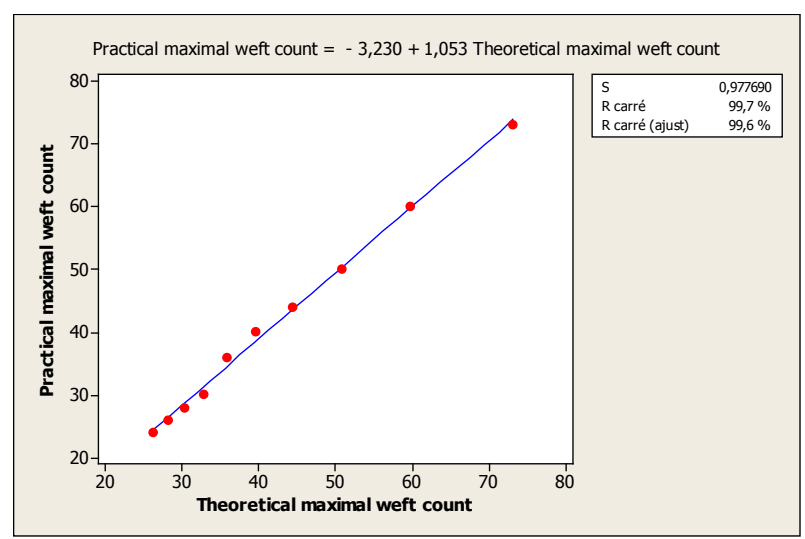

Air jet weaving machine-Polyester Weft

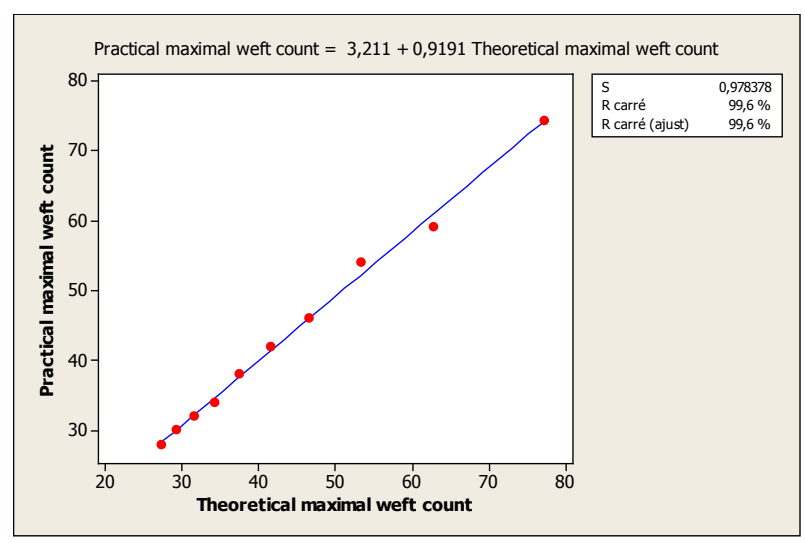

Rapier weaving machine-Cotton Weft

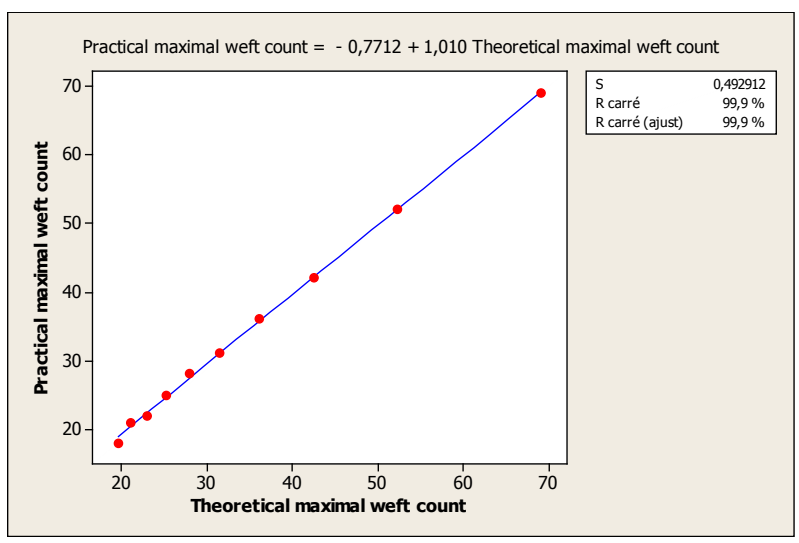

Projectile weaving machine-Cotton Weft

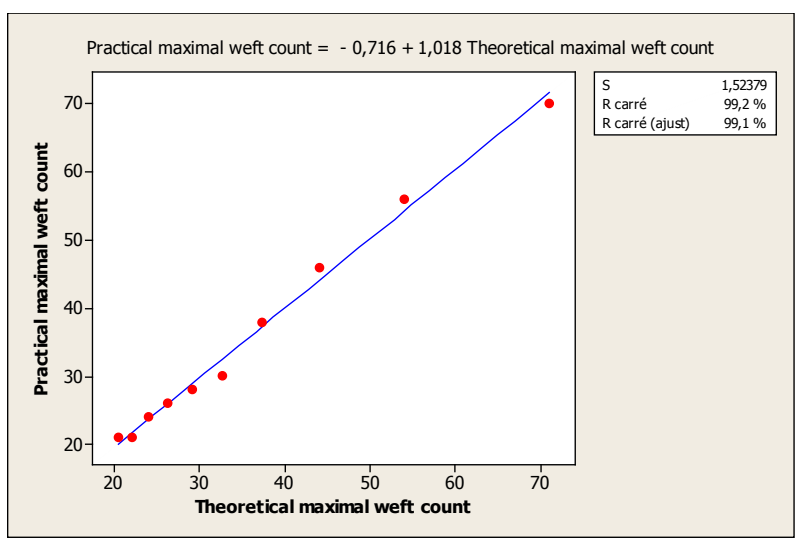

Air jet weaving machine-Cotton Weft

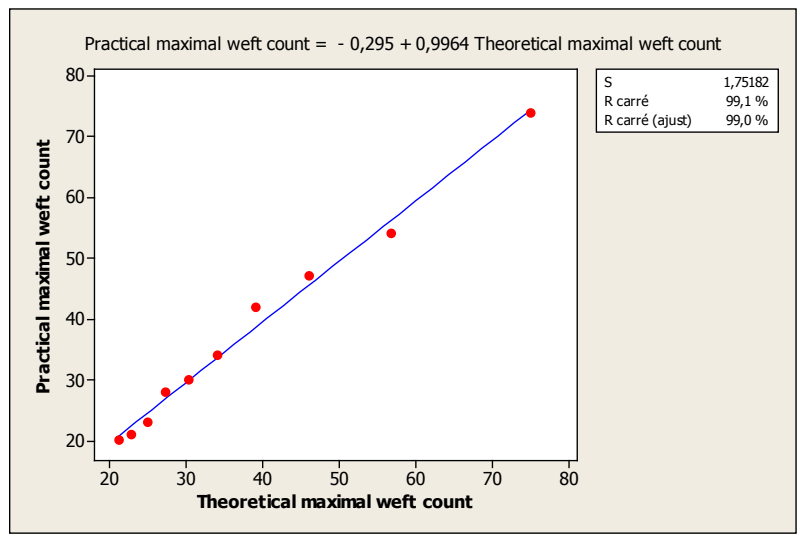

Figure 7. Correlation between practical and theoretical maximal weft count for simple weaves

And Very good correlation (Figure 9) is observed between practical and theoretical maximal weft count for complex weaves. The practical weft count is obtained by experience at the weavability limit using different looms and the theoretical maximal weft count is obtained by using the new availability-limit relationships.

\subsection{Confirmation of the reviewing existing equations}

The table 2 shows, for existing samples of woven fabrics that we have analysed in our laboratory, that if we rely on the old relationships, the samples of woven fabrics should not exist and they are judged no-weavable fabrics 
Rapier weaving machine-Polyester Weft

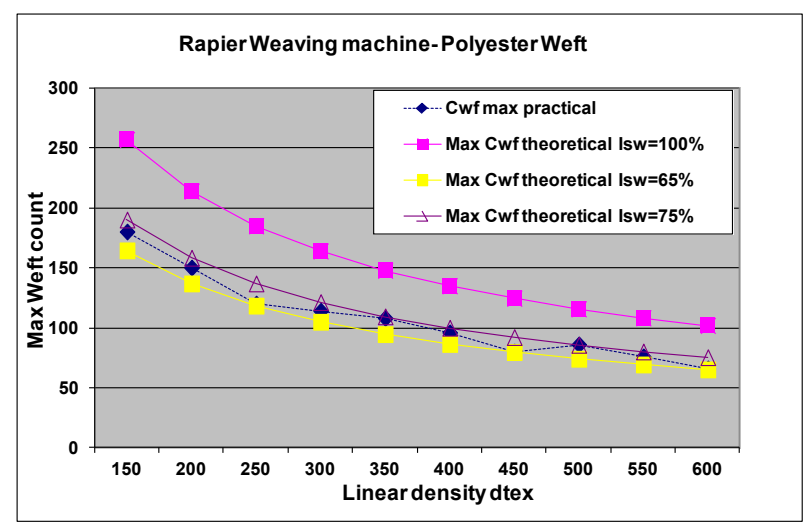

Projectile weaving machine-Polyester Weft

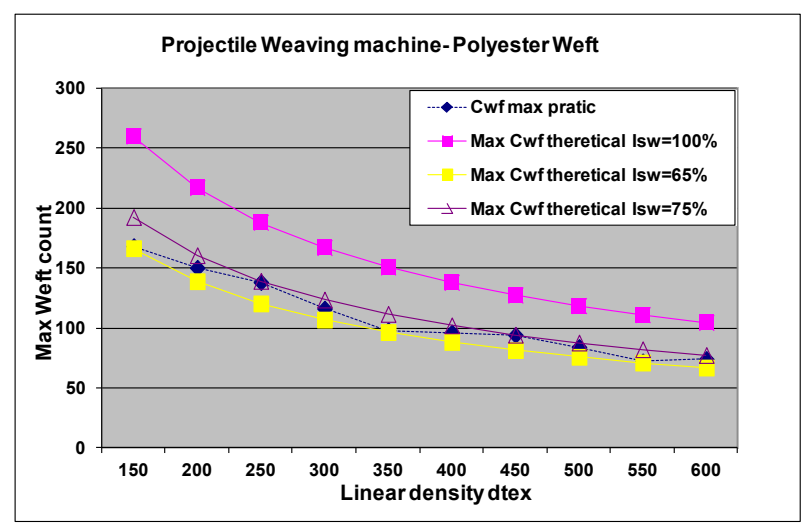

Air jet weaving machine-Polyester Weft

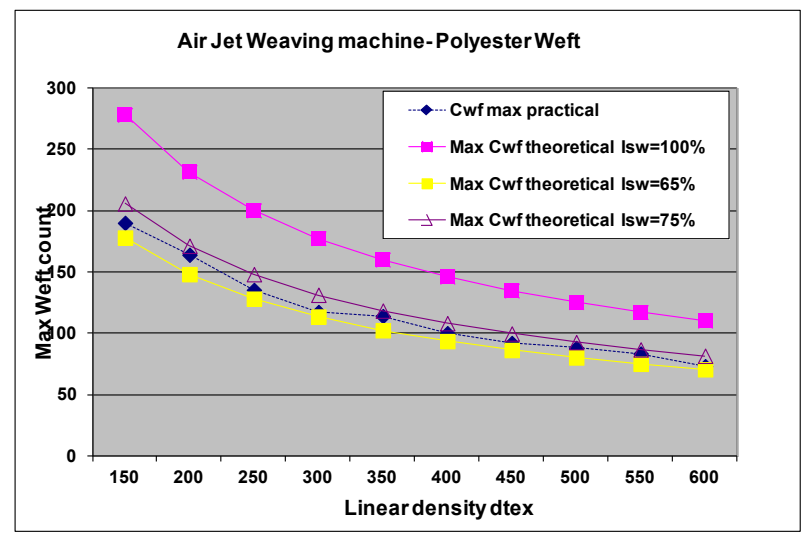

Rapier weaving machine-Cotton Weft

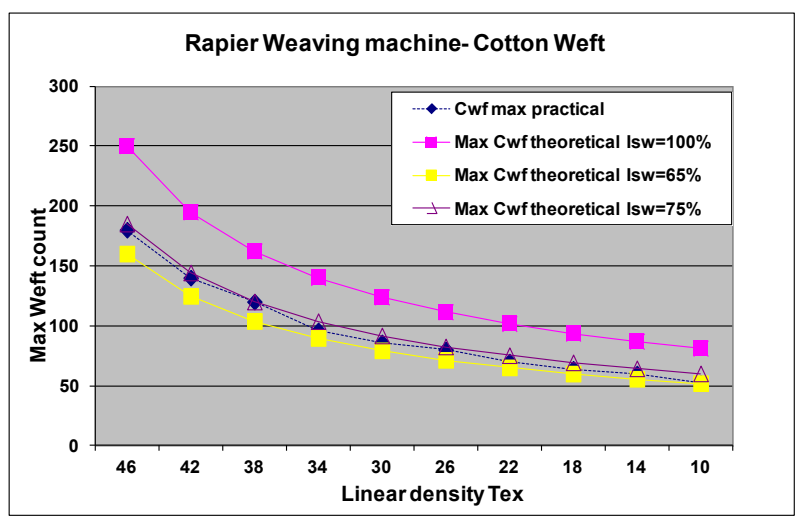

Projectile weaving machine-Cotton Weft

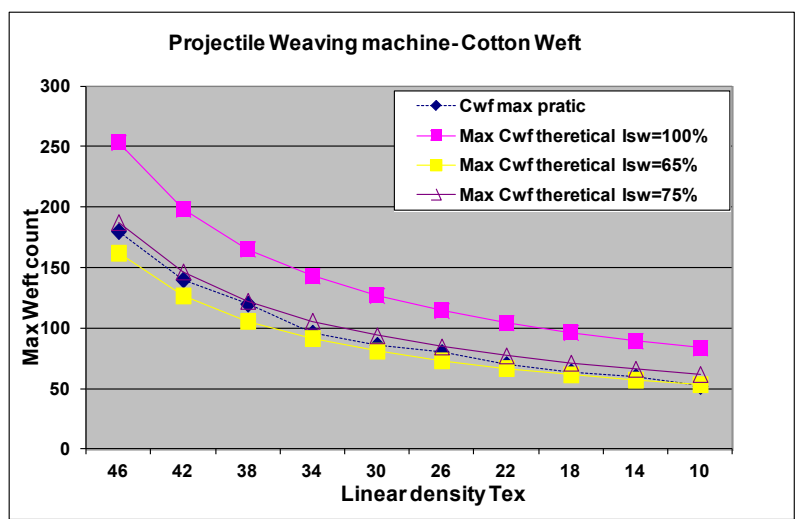

Air jet weaving machine-Cotton Weft

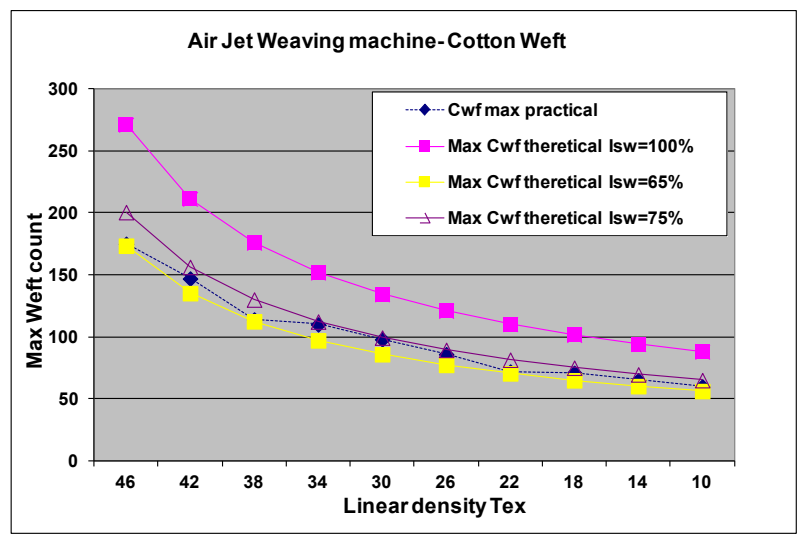

Figure 8. Validation of the Weavability-limit relationships for complex weaves

\section{The weavability old relationships index exceed $72 \%$}

Against the new relationships confirms the existence and accurately informs us about their tightening construction and their weavability.

The weavability new relationships index does not exceed $72 \%$

\section{Conclusion and future work}

The theoretical relationships were examined and found to agree with experimental results. It was concluded that the theoretical relationships and the graphs derived from them were a useful tool for weavers in predicting the weavability of a proposed fabric construction. 
Rapier weaving machine-Polyester Weft

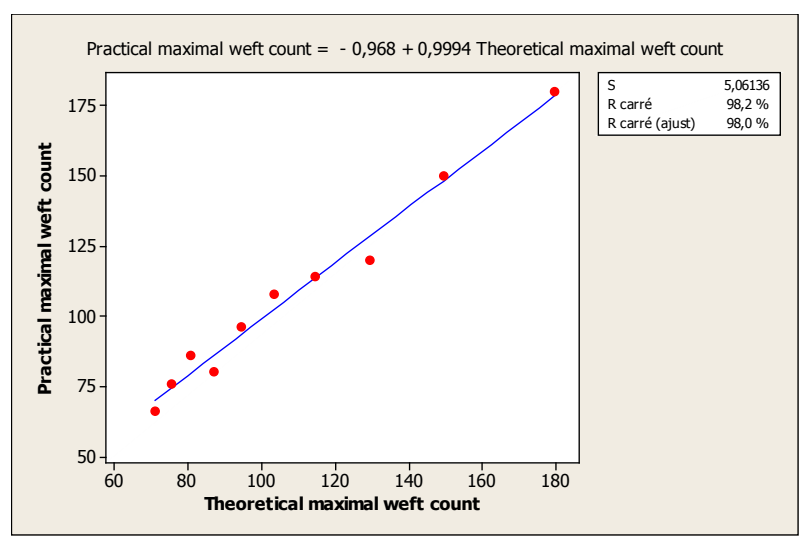

Projectile weaving machine-Polyester Weft

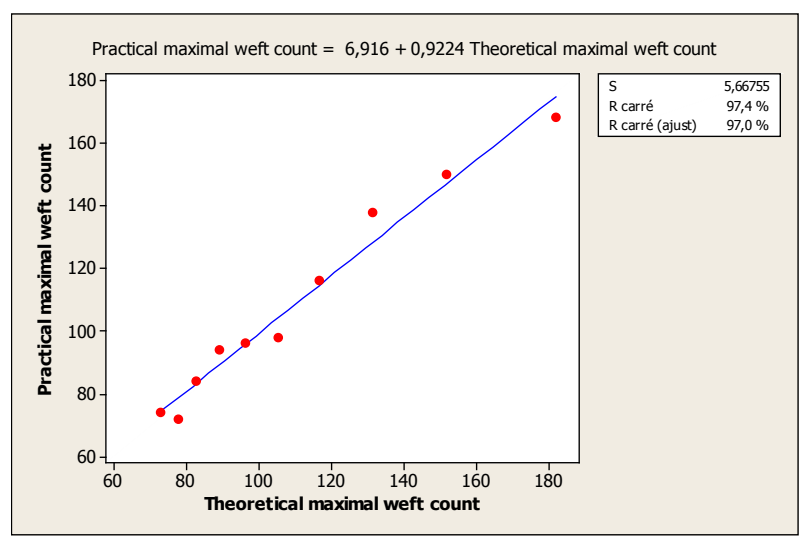

Air jet weaving machine-Polyester Weft

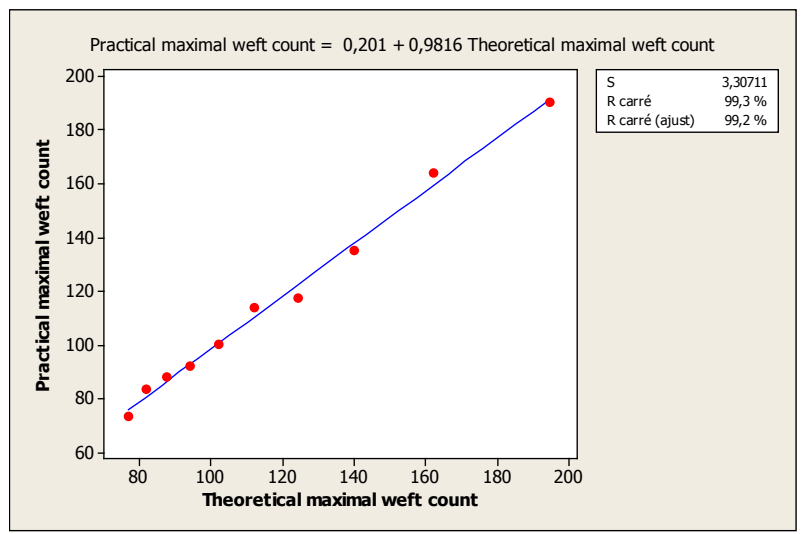

Rapier weaving machine-Cotton Weft

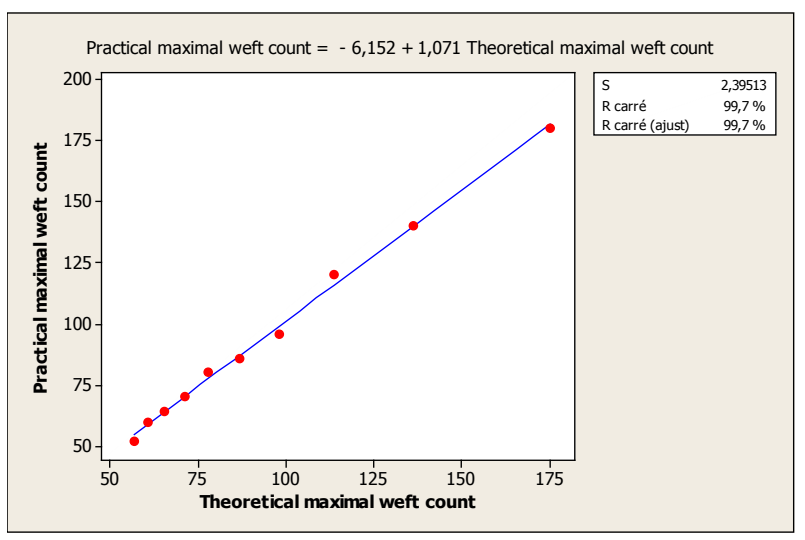

Projectile weaving machine-Cotton Weft

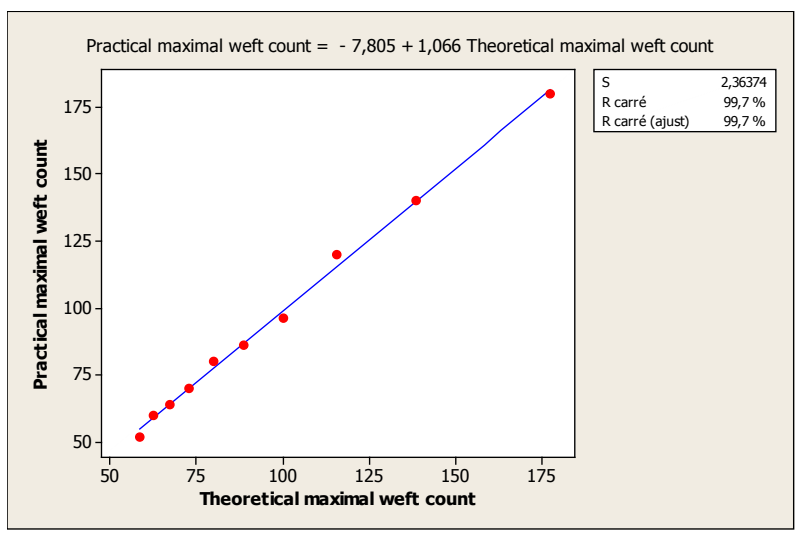

Air jet weaving machine-Cotton Weft

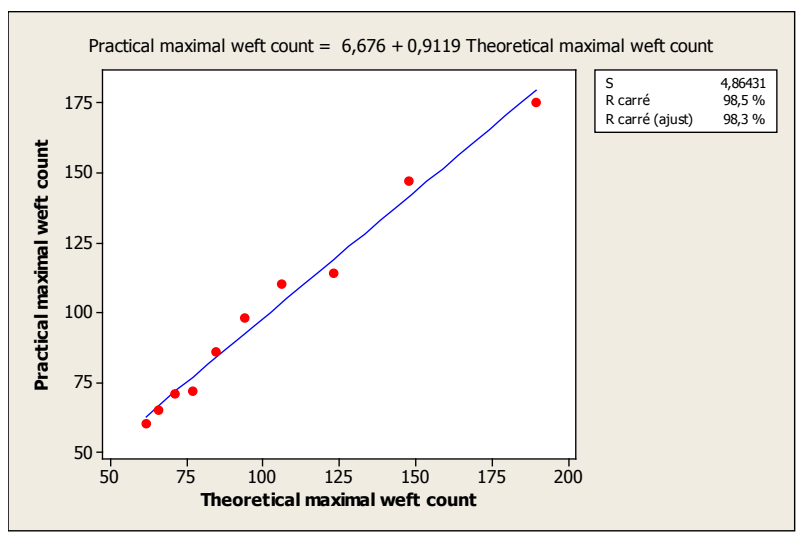

Figure 9. Correlation between practical and theoretical maximal weft count for complex weaves

The weavability limit and tightness relationship, that has just been defined, presents a certain number of substantial advantages:

- They are universally used

- They are independent of the weaving machine used;

- They allow the comparison of different woven fabrics;

- They allow an optimal choice of thread counts 
Table 2. Confirmation of the reviewing existing equations

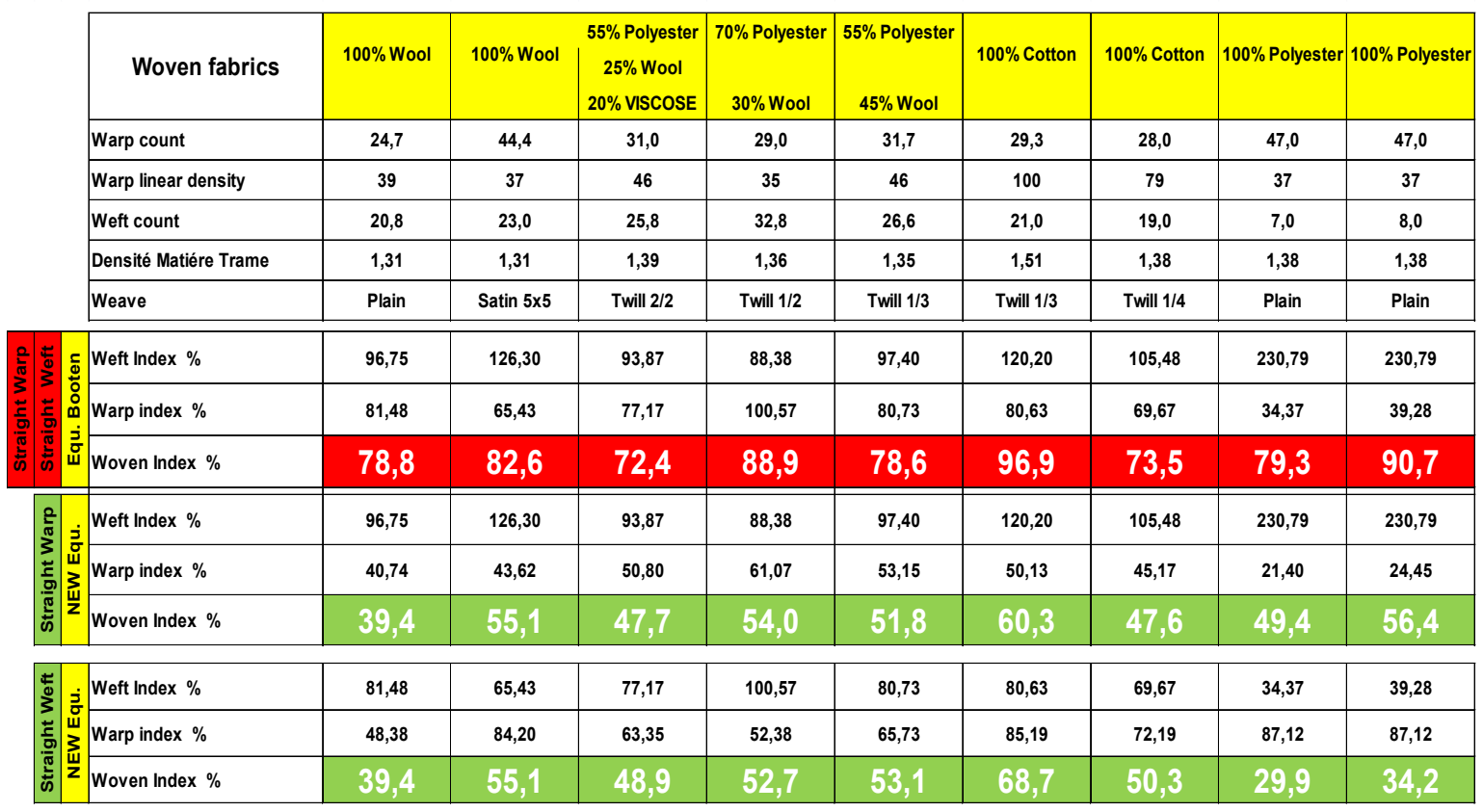

\section{References}

[1] D.P. Adams, E.R. Schwarz, and S. Backer. The Relationship between the Structural Geometry of a Textile Fabric and its Physical Properties. PartVI: Nomographic Solution or the Geometric Relationships in Clolh Geometry. Text. Res. J., 1956, 26, 653-665.

[2] M.W. Alford. Ratio Comparison of Fabric Structures. J. Text. Inst., 1964, 55, T83-98.

[3] T.R. Ashenhurst. A treatise on textile calculations and the Structure of Fabrics, 1884.1 Broadbent, London.

[4] N.C. Gee. Cloth Setting and Setting Theories. Text. Mfr, 1953, 80, 381-384.

[5] N.C. Gee. Cloth Setting and Setting Theories. Text. Mfr, 1953, 80, 399-401.

[6] H.C. Haller. Maximum Construction Tables tor Fabrics of Creslan Acrylic Fiber and Other Fibers. Paper presented at National Meeting of AATCC, Philadelphia, PA, USA, 1960.

[7] J.B. Hamilton. A Direct Method tor Measuring Yam Diameters and Bulk Densities under Conditions of Thread Flattening. J. Text. Inst., 1959,50, T655-T672.

[8] H.W. Russell. Help for Designers. Construction Factor - An Aid to FabJic Evaluation and Design. Text. Industr 1965,129, No. 6, 51-53.

[9] S. Brierley. Theory and Practice of Cloth Setting. Text. Mfr, 1931, 58, 3-4.

[10] S. Brierley. Theory and Practice of Cloth Setting. Text. Mfr, 1931, 58, 47-49.

[11] S. Brierley. Cloth Setting Reconsidered. Part I. Tex. Mfr, 152, 79, 349-351.

[12] S. Brierley. CIOUI Setting Reconsidered. Part II. Text. Mfr. 1952. 79, 431-433.

[13] S. Brierley. Cloth Setting Reconsidered. Part III. Text. Mfr. L952, 79, 449-453.

[14] S. Brierley. Cloth Setting Reconsidered. Part IV. Text. Mfr, L952, 79, 533-537.
[15] H. Bogaty, G.H. Lourigan, and H.E. Hanis. Structural Compactness of Woven Wool Fabrics and their Behavior in Modern Washing Machines. Text. Res. J., 195.8., 28, 733-737.

[16] J.J. Brown and R.A. Rusca. the Effect of Fabric Structure on Fabric Properties. Text. Res. J., 1955.5, 25,472-476.

[17] K.J. Butler and W.T. Cowhig. Yam In'egularity Picture Recorder. Skinner 's Silk \& Rayol1 Rec., L954, 28, 11781181.

[18] H. Catling. Some Effects of Sinusoidal Periodic Yam Thickness Variation on the Appearance of Woven Cloth. J. Text. Insl., 1958, 49, T232-T246.

[19] N.H. Chamberlain and D.C. Snowden. Loom Study by Means of the Cathode Ray Oscillograph. Part I: Variation in Individual Warp Thread Tension during the Weaving Cycle. J. Text. Inst., I.2.48, 39, T23-T43.

[20] J.B Dickson. Practical Loom Experience on Weavability Limits. Text. Res. J., 1948. 24. 1083-1093.

[21] P. Ellis and D.L. Munden. ATheoretical Analysis and Experimental Study of the Plain Square Weave. Part I: The Effect of Seu and Degree of Relaxation on the Measured Cross-sectional Dimensions of Yarns.J. Text.inst., 1973, 64, 279-294.

[22] R. Foster. Weaving Investigations Periodic Patteming in Fabrics. J. Text. Inst., L952, 43 P742-P754.

[23] S. Galuszynski. Fabric Tightness: A Coefficient to Indicate Fabric structure. J. Text. Inst., 981 72,44-49.

[24] J.B. Hamihon. A General System of Woven Fabric Geometry. J. Text. Inst., 1964,55, T66-T82.

[25] J. W.S. Hearle, P. Grosberg, and S. Backer. Structural Mechanics of Fibers. Yarns and Fabrics, Vol. I, Wiley Interscience, New York, NY, USA, 1969.

[26]E.R. Kaswell. Textile Fibers, Ya/'I1s. and Fabrics: A Comparative Survey of Their Behavior with Special Reference to Wool, Reinhold, New York, NY, USA, 1953. 
[27] Kemp. An Extension of Peirce's Cloth Geometry to the Treatment of Non-circular Threads. J. Text. Inst., 1958, 49, T44-T47.

[28] W. Law. A Practical Treatise on Cloth Building. Wool Rec., 1922, 21, 968 et seq. (series concluding on 1486).

[29] L. Love. Graphical Relationships in Cloth Geomelty for Plain, Twill, and Sateen Weaves. Text. Res. J., 1954, 24, 1073-1083.

[30] Newton. The comparison of Woven Fabrics by Reference to their Tightness. J. Text. Inst., 1995, 86,232-240.

[31] Newton. Tightness Comparison of Woven fabrics. Indian Text. J. 1991, 101, Feb., 38-40.

[32] E. V. Painter. Mechanics of Elastic Performance of Textile Materials. Part VIII: Graphical Analysis of Fabric Geometry. Text. Res. J.. 1952, 22, 153-169,
[33] F.T. Peirce. The Geometry of Cloth Structure. J. Text. Inst., 1937, 28, T45-112.

[34] D.E.A. Plate and K. Hepworth. Beat-up Forces in Weaving. Part II. J. Text. Inst., 1973, 64,233-249.

[35] E.B. Sehuler. Analysis of Loom Stoppages. M.S. Thesis, North Carolina State University, 1993.

[36] A.M. Seyam. On the Mechanics of Woven Fabrics. Doctoral Thesis, North Carolina State University, 1985.

[37] A.M. Seyam. Weavability Limit of Yarns with Thickness Variation in Shuttleless Weaving: The Single Weft Yarn Feeder Case. Text. Res. J., 2000, 70, 129-134.

[38] A.M. Seyam and A. El-Shiekh. Mechanics of Woven Fabrics. Part 1: Theoretical Investigation of Weavability Limit of Yarns with Thickness Variation. Tex. Res. J., 1990, 60,389-404. 